\title{
A LATTICE FIXED-POINT THEOREM WITH CONSTRAINTS ${ }^{1}$
}

\author{
BY ALVIN E. ROTH
}

Communicated by S. Eilenberg, June 11, 1974

This paper presents a lattice fixed-point theorem having applications in game theory and elsewhere. The results presented here form part of the author's Ph.D. thesis in Operations Research, conducted under the supervision of Robert Wilson.

Let $L$ be a complete lattice. Denote elements of $L$ with small letters $a, b, c, \cdots$, and denote subsets of $L$ with capital letters $A, B, C \cdots$. Consider a function $U: L \rightarrow L$ with property $\mathrm{P}$ : for any $A \subseteq L, U(\backslash A)=$ $\wedge U(A)$, where $U(A) \equiv\{U(a) \mid a \in A\}^{2}{ }^{2}$ Denote the composition of $U$ with itself by $U^{2}$.

Property $\mathbf{P}$ implies $U^{2}(b)$.

LEMMA 1. (1) $a \leqslant b$ implies $U(a) \geqslant U(b) ;(2) a \leqslant b$ implies $U^{2}(a) \leqslant$

If we define $L_{D}(U) \equiv\{a \in L \mid a \leqslant U(a)\}$ and $L_{D}\left(U^{2}\right) \equiv\{a \in L \mid a \leqslant$ $\left.U^{2}(a)\right\}$, then we have

LEMMA 2. (1) $U^{2}: L_{D}(U) \rightarrow L_{D}(U)$; (2) $U^{2}: L_{D}\left(U^{2}\right) \rightarrow L_{D}\left(U^{2}\right)$.

LEMMA 3. $L_{D}\left(U^{2}\right)$ is a complete join subsemilattice of $L$.

We know from Tarski's theorem [1] that $U^{2}$ has a fixed point in $L$, while it is not generally true that $U$ has a fixed point. We can, however, state the following

THEOREM. There exists an element $s \in L$ such that $s=U^{2}(s)$ and $s \leqslant U(s)$.

AMS (MOS) subject classifications (1970). Primary 06A23, 90D12; Secondary 05 C99.

Key words and phrases. Complete lattice, fixed-point, stable set.

1 This work was partially supported by National Science Foundation Grant GS-40104 at the Institute for Mathematical Studies in the Social Sciences, Stanford University, and partially by AEC Contract AT(043)-326 PA \#18 at the Department of Operations Research, Stanford University.

2 Join and meet are represented by $\vee$ and $\Lambda$. 
Proof. Let $D=L_{D}(U) \cap L_{D}\left(U^{2}\right) . D$ is nonempty since inf $L \in D$. Let $M$ be a maximal chain in $D$, and let $s=\bigvee_{M}$. Then $s \in L_{D}\left(U^{2}\right)$ by Lemma 3.

Suppose $s \notin U(s)$. By property $\mathrm{P}, U(s)=\bigwedge U(M)$, so $s \notin U(s)$ implies the existence of an $m \in M$ such that $s \notin U(m)$. Consequently there exists $n \in M$ such that $n \nless U(m)$. Now $M \subseteq L_{D}(U)$ implies $m \leqslant U(m)$ and $n \leqslant U(n)$. Furthermore, $M$ is a chain, so either $m \leqslant n$ or $n \leqslant m$. But $n \nless m$, since $n \nless U(m)$. And, by Lemma $1, m \leqslant n$ implies $U(m) \geqslant$ $U(n) \geqslant n$, which gives a contradiction. Therefore $s \leqslant U(s)$; i.e., $s \in L_{D}(U)$.

By Lemma $2, U^{2}(s) \in D$. Also, $s \in D$ implies $s \leqslant U^{2}(s)$. If $s<U(s)$, then the chain $M$ is not maximal, contrary to assumption. So $s=U^{2}(s)$. Q.E.D.

N.B. The element $s$ produced above is maximal in $D$. A sufficient condition to insure $s \neq \inf L$ is that there exists an $m \in D$ such that $m>\inf L$.

To see how functions $U$ with property $P$ arise, consider an arbitrary set $X$ on which is defined an arbitrary binary relation $>$. For each $x \in X$, define $\operatorname{Dom}(x)=\{y \in X \mid x>y\}$; and for each $S \subseteq X$, define $\operatorname{Dom}(S)=$ $\bigcup_{x \in S} \operatorname{Dom}(x)$. Let $U(S)=X-\operatorname{Dom}(S)$, and let $L=2^{x}$ be the complete lattice of subsets of $X$ ordered by set inclusion. Then the function $U$ : $L \rightarrow L$ has property $P$. Therefore we can state the following

COROLlary. There exists a subset $S \subseteq X$ such that $S=U^{2}(S)$ and $S \subseteq U(S)$

In particular, we can take $X$ to be the set of imputations of a cooperative game, and $>$ to be the domination relation. A set $S$ thus produced generalizes and closely resembles a von Neumann-Morgenstern solution, which is a fixed point of $U$, and which fails to exist for some games. Lucas [2] produced a game for which no solution exists, but for which the zet $S$ discussed here can be interpreted in the manner usually associated with solutions. This will be studied in detail in subsequent publications.

\section{REFERENCES}

1. G. Birkhoff, Lattice theory, 3rd ed., Amer. Math. Soc. Colloq. Publ., vol 25, Amer. Math. Soc., Providence, R. I., 1967. MR 37 \#2638.

2. W. F. Lucas, A game with no solution, Bull. Amer. Math. Soc. 74 (1968), 237-239. MR 36 \#3581.

3. A. E. Roth, Subsolutions of cooperative games, Technical Report no. 118 , Institute for Mathematical Studies in the Social Sciences, Stanford University; also: 
Technical Report no. 73-12, Department of Operations Research.

4. - A fixed-point approach to stability of cooperative games, Fixed Points: Algorithms and Applications, Karamardian \& Garcia (editors), Academic Press (forthcoming).

5. - Topics in cooperative game theory, Technical Report SOL 74-8, Systems

Optimization Laboratory, Department of Operations Research, Stanford University, July 1974.

DEPARTMENT OF OPERATIONS RESEARCH, STANFORD UNIVERSITY, STANFORD, CALIFORNIA 94305

Current address: Department of Business Administration, Department of Economics, University of Illinois, Urbana, Illinois 61801 